\title{
Perspective and agency during video gaming influences spatial presence experience and brain activation patterns
}

\author{
Michael Havranek ${ }^{1 *}$, Nicolas Langer ${ }^{2}$, Marcus Cheetham ${ }^{2}$ and Lutz Jäncke ${ }^{2}$
}

\begin{abstract}
Background: The experience of spatial presence (SP), i.e., the sense of being present in a virtual environment, emerges if an individual perceives himself as 1 ) if he were actually located (self-location) and 2) able to act in the virtual environment (possible actions). In this study, two main media factors (perspective and agency) were investigated while participants played a commercially available video game.
\end{abstract}

Methods: The differences in SP experience and associated brain activation were compared between the conditions of game play in first person perspective (1PP) and third person perspective (3PP) as well as between agency, i.e., active navigation of the video game character (active), and non-agency, i.e., mere passive observation (passive). SP was assessed using standard questionnaires, and brain activation was measured using electroencephalography (EEG) and SLORETA source localisation (standard low-resolution brain electromagnetic tomography).

Results: Higher SP ratings were obtained in the 1PP compared with the 3PP condition and in the active compared with the passive condition. On a neural level, we observed in the 1PP compared with the 3PP condition significantly less alpha band power in the parietal, the occipital and the limbic cortex. In the active compared with the passive condition, we uncovered significantly more theta band power in frontal brain regions.

Conclusion: We propose that manipulating the factors perspective and agency influences SP formation by either directly or indirectly modulating the ego-centric visual processing in a fronto-parietal network. The neuroscientific results are discussed in terms of the theoretical concepts of SP.

Keywords: Spatial presence, Brain activation, Perspective, Agency, EEG, LORETA, Fronto-parietal network, Posterior parietal cortex, Premotor cortex

\section{Background}

When confronted with well-designed virtual reality (VR) scenarios, many people experience a subjective sense of actually being in the VR environment while transiently being unaware of the technology that delivers the stream of virtual input to the senses. This specific feeling has been coined "spatial presence" (SP) [1-3]. This definition of SP emphasises the important role of spatial cues and the subjective strategy involved in recruiting attentional resources for processing sensory input (perceptionoriented approach). An alternative view has been

\footnotetext{
* Correspondence: m.havranek@bli.uzh.ch

${ }^{1}$ Clinic for Affective Disorders, University Clinic of Psychiatry Zurich, Zurich, Switzerland

Full list of author information is available at the end of the article
}

proposed by Sanchez-Vivez and Slater [4]. They underline the particular contribution of supported actions in the real or virtual environment (VE) as a constituent feature of the experience of reality. They argue that the sense of "being there" in a VE is strongly grounded on the ability "to do there" (action-oriented approach).

In the context of a more recent theoretical paper, both approaches are combined and extended [5]. In this theoretical account, a psychological construct referred to as "egocentric reference frame" (ERF) is used to explain SP. An ERF is a mental model of the world organised from a first-person perspective and contains information about the spatial properties of the immediate surroundings. A mediated environment (e.g. VR) offers an alternative ERF to the users' real-world ERF. The sense of SP 
emerges if this alternative ERF is chosen as the primary ERF over the competing ERF of the real physical world. The outcome of this selection process is considered to be based on two critical questions: whether a person perceives himself 1 ) as if actually located in the mediated environment (self-location) and 2) as being able to act in the mediated environment (possible actions).

Only a few studies to date have examined the neural underpinnings of SP. For example, Baumgartner et al. $[6,7]$ identified a distinct network of fronto-parietal brain regions involved in the generation and regulation of SP during exposure to virtual roller coaster scenarios. Using EEG [6], they found activations in parietal brain regions and deactivations in frontal brain regions in participants experiencing high SP compared with participants experiencing low SP. They concluded from their findings that spatial cues presented in the roller coaster scenario generate a sense of SP by strongly activating parietal brain regions (in the dorsal visual stream particularly) that thus lead to enhanced egocentric processing of the displayed environment. In a subsequent fMRI study [7], they concluded that frontal (de)activations relating to differences in SP are closely associated with modulatory processes in the dorsolateral prefrontal cortex (DLPFC). Specifically, they concluded that activation in the DLPFC modulates the sense of SP by down-regulating egocentric visual processing in the dorsal visual stream, as reflected in those participants who experience low SP. This interpretation has received support by further studies using EEG [8] and transcranial direct current stimulation (tDCS) [9], the latter relating these ideas to neuroscientific concepts of behaviour and impulse control [for review, see [10]], and by an interesting study investigating flow experience during video game playing [11].

Considering the increasing importance of VR in our society (e.g. in TV, video gaming and the internet), this study aimed to extend our understanding of SP by investigating the neural correlates of the theoretically proposed key stages in the formation of SP. It was decided to experimentally influence the choice of the virtual ERF as the primary ERF by manipulating the impression of self-location on the one hand and the perceived possible actions on the other hand. To do so, the two important media factors perspective (first-person perspective vs. third-person perspective) and agency (being the agent of the actions in the VR vs. being the observer of the actions in the VR) were experimentally controlled and manipulated. These two factors have been shown to influence experience of SP in various psychometrical and behavioural studies [12,13]; however, no study has investigated their influence on SP on a neural level. Thus, the present study sought to establish how manipulating the factors perspective and agency would influence the impression of self-location and the perceived possible actions and the formation of SP on a perceptual and a neural level.

To investigate this, electroencephalography (EEG) was recorded and subjective SP experience was assessed while participants played the commercially available video game "The elder scrolls 4: Oblivion". Participants had to play a simple, non-arousing task, in which they were required to navigate the video game's avatar (i.e. the virtual character) to a given location in four different conditions (i.e. in a $2 \times 2$ design combining the factors perspective and agency). The two perspective conditions entailed playing in the $1 \mathrm{PP}$ or in the 3PP. The two agency conditions allowed participants to either actively control the avatar (active) or to passively observe the avatar while it was in fact controlled by another player (passive). EEG registration was chosen over fMRI measurements to facilitate presentation of a real-life gaming situation and because EEG has been shown to be a valuable tool in the study of video and computer gaming in previous research $[14,15]$. In order to directly compare our EEG results with the previously obtained functional magnetic resonance imaging (fMRI) and tDCS findings of our group $[7,9,10]$, the sLORETA software (standard low-resolution brain electromagnetic tomography) was used also in order to calculate the cortical sources [16]. Based on previous studies, it was hypothesized that manipulating the factors perspective and agency influences SP by either directly or indirectly modulating the egocentric processing in the dorsal stream. The following predictions were thus formulated for our EEG experiment:

$\mathrm{H} 1$ : Playing the video game under 1PP compared with 3PP will enhance the experience of SP and reveal stronger activation in parietal areas (especially in the dorsal visual stream).

$\mathrm{H} 2$ : Actively playing the video game compared with passively observing it will enhance the experience of SP and show stronger deactivation in frontal brain areas (e.g. in the DLPFC).

\section{Methods}

\section{Participants}

Twenty healthy male volunteers took part in this experiment (age $=19-32$ years; $M=23.5$ years; $S D=3.83$ ). One person had to be excluded from statistical analysis due to missing EEG data. All participants were consistently right-handed and native speakers of German. Handedness was assessed using the Annett-Handedness Questionnaire [17]. To measure health status, a questionnaire regarding neurological and psychiatric health, drug use, hearing and visual deficits was used. None of the participants reported any history of neurological or psychiatric disease, sensory impairments or subjective cognitive 
impairments. To control for any effects of gaming expertise, the sample was stratified according to the gaming experience of the participants into 10 experienced gamers ( $>5$ hours per week, $M=11.70$ and $S D=8.24$ ) and 10 non-gamers $(<1$ hour per week, $M=0.2$ and $S D=0.42$ ). The participants were recruited from various sources, such as the university, the social network of the investigators and via internet ads. The local ethics committee approved the study and all participants gave written informed consent.

\section{Experimental task}

Participants played a specific video game scenario from the commercially available video game "The elder scrolls 4: Oblivion" (Bethesda Softworks LLC, a Zeni Max Media Company). The game was played on the Playstation 3 console (Sony Corporation) using the original Playstation 3 controller, on a TV screen ( 26 inch) placed at a distance of $1.5 \mathrm{~m}$ in front of them. "Oblivion" is a single-player role-playing game in which the player has the ability to explore a richly detailed game world using a realistic looking human avatar (see Figure 1). Because all participants played this game for the first time, a simple scenario was used in which they were required to navigate their avatar to a given location. The selected game scenario was non-arousing, without enemies or other dangerous situations. A $2 \times 2$ factorial design with the factors perspective and agency was used. After a short training session to learn the correct navigation of the avatar, each person performed two simple tasks. In the active condition, participants were instructed to complete a mission in which they had to navigate their avatar to follow a specific path and to find the entry of a castle. In the passive condition, participants were instructed to closely pursue how the avatar completed the same task but controlled by another player. These tasks were performed by all participants from two different perspectives. In the 1PP, participants performed the task by looking through the eyes of the avatar, that is, as if actually located in its body. In the 3PP, participants performed the task from a viewpoint behind and above the avatar (see Figure 1). Thus, there were 4 conditions (1PP-active, 1PP-passive, 3PP-active and 3PP-passive) each of which was performed once by each participant. Each trial took 2-3 minutes, depending on the speed of each participant in the active conditions. The 4 trials were systematically randomised. During these trials, continuous EEG was recorded. After each trial, participants answered a short questionnaire about their SP experience (see next paragraph).

\section{Psychometrical measurements}

Since the sense of SP is a subjective feeling, the MECSPQ questionnaire was used [18]. The MEC-SPQ is
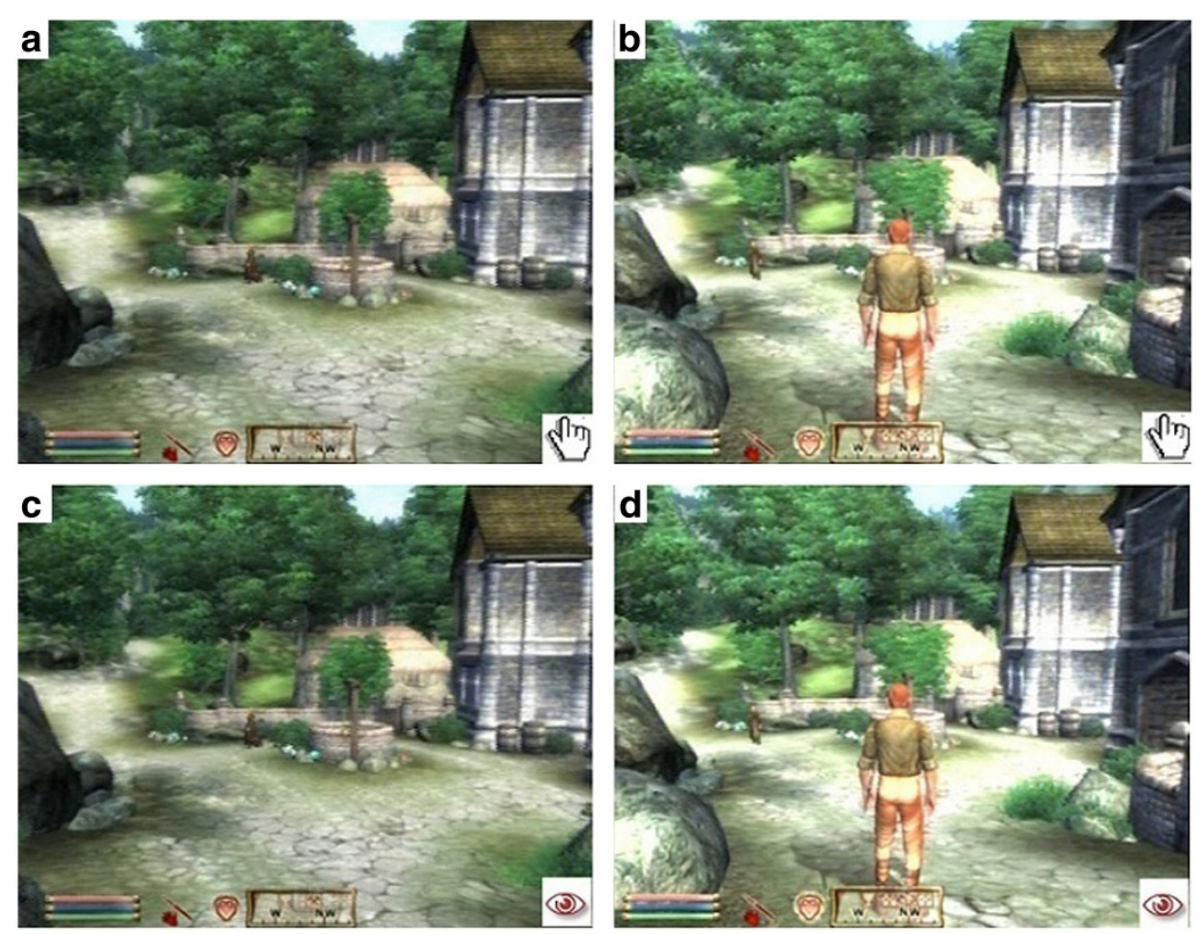

Figure 1 2x2 factorial design: Playing in the 1PP was compared with playing in the 3PP. This was further examined in relation to actively playing and controlling the video game avatar or simple passive observation of the avatar controlled by another player. These 4 conditions were: 1PP-active (Figure 1a), 3PP-active (Figure 1b), 1PP-passive (Figure 1c) and 3PP-passive (Figure 1d). 
specifically designed to measure SP and consists of 9 scales. Four of these cover process factors (attention allocation, spatial situation model, self-location, possible actions), two scales refer to states and actions (higher cognitive involvement, suspension of disbelief) and three scales address more trait-like personality characteristics (i.e. domain specific interest, visual spatial imagery, and absorption). Two of the process scales are specifically intended to reflect physical (or spatial) presence: namely the self-location and the possible actions scale. The subscale self-location measures the impression of the subject to be physically in the middle of the virtual game environment (e.g. "I felt like I was actually there in the environment of the presentation."). The sub-scale possible actions assesses the impression of being able to act in the VE (e.g. "The objects in the presentation gave me the feeling that I could do things with them."). There are three versions of the MEC-SPQ: a full, a medium and a short version of all the scales (with 8,6 or 4 items per scale). All versions have been validated in large samples in different cities within three countries (Los Angeles, Helsinki, Porto) [18]. In our study, a German translation of the 4-item scale for the sub-scales self-location and possible actions was used. Participants were instructed to answer on an 8- point scale ranking (" $0=\mathrm{I}$ do not agree at all" to "7 = I agree fully"), instead of the proposed 5point scale because it was determined in the pilot study that more precise results would be derived from the use of a larger scale. In the statistical analysis, the Friedman test was used as a first step to identify effects of the factors perspective and agency on the sub-scales selflocation and possible actions. In a second step, the Wilcoxon test was used to make three pairwise comparisons between the four conditions (1PP-active vs. $3 \mathrm{PP}$-active, 3PP-active vs. $1 \mathrm{PP}$-passive and 1PP-passive vs. 3PP-passive). The results were considered significant in case of $p<0.05$, corrected for multiple comparisons (Bonferroni).

\section{EEG recordings and preprocessing}

EEG was recorded from 30 scalp electrodes using a Brain Vision amplifier system (Brain Products, Germany). Silver-silver-chloride electrodes were used in association with the "Easy Cap System"(International 10-10 system, Falk Minow Services, Germany) and were attached according to the international 10-10 system in the following positions Fp1/2, F3/4, F7/8, Fz, FCz, FT7/8, FC3/4, T7/8, C3/4, Cz, TP7/8, TP9/10, CP3/4, CPz, P7/8, P3/4, $\mathrm{Pz}, \mathrm{O} 1 / 2$ and $\mathrm{Oz}$. Recording reference was at $\mathrm{FCz}$, with off-line re-referencing to average reference $[19,20]$. An electro-oculogram (EOG) was recorded from two additional electrodes placed below the outer canthus of each eye to record horizontal eye movements. Brain Vision Recorder and Analyzer (Brain Products, Germany) were used to record and analyze the data. The digital sampling rate was $500 \mathrm{~Hz}$; impedance was kept below $10 \mathrm{k} \Omega$. Participants were seated in a comfortable chair with their head resting in a head mounting within a sound- and electrically shielded chamber with dim illumination. In the beginning of the experiment, a short 3 minute open eye resting condition EEG was taken as baseline recording. Then, after a short training session (to learn the navigation of the avatar), continuous EEG was recorded during each experimental trial.

After recording EEG, the data were bandpass filtered from 1 to $30 \mathrm{~Hz}$ using a notch filter at $50 \mathrm{~Hz}$. Then, an independent component analysis (ICA) algorithm provided in the EEGlab software was used to correct for artefacts (e.g. eye blinks \& eye movements). It has been shown that ICA can effectively detect, separate and remove activity in EEG records from a wide variety of artificial sources [21]. In addition, all recorded data were carefully and individually checked for artefacts (sweating \& muscle artefacts) by visual inspection. The artefactfree EEG was recomputed against the average reference [22]. Each of the four conditions was then segmented into epochs of 2-s duration. We used 68 segments per subject and condition for all the analyses $(30.88 \%$ of all epochs were excluded).

\section{Scalp map analysis}

A discrete Fourier transformation algorithm was applied to all 2-s epochs according to the frequency band spectra defined by Kubicki et al. [23]: delta $(1.5-6 \mathrm{~Hz})$, theta (6.5-8 Hz), alpha1 (8.5-10 Hz), alpha2 (10.5-12 Hz), beta1 $(12.5-18 \mathrm{~Hz})$, beta2 $(18.5-21 \mathrm{~Hz})$, beta3 (21.5$30 \mathrm{~Hz})$ and omega $(1.5-30 \mathrm{~Hz})$. Of these frequency band spectra, the alpha and theta band power interested us the most. Alpha band power has repeatedly been shown to be inversely related to haemodynamic brain responses especially in lateral frontal and parietal brain areas [24-26] and based on these results, we take the alpha band power as an indicator of cortical deactivation in these areas. Theta rhythm in frontal areas is referred to as "frontal midline theta" (FM-theta) and has been observed during a large variety of tasks such as mental calculation, working memory and learning, error processing, and meditation $[27,28]$. Therefore, FM-theta has been interpreted as a correlate of heightened mental effort and sustained attention required during a multitude of operations. Furthermore, FM-theta has been found in previous EEG based video gaming studies [29-31]. Thus, alpha band power (including alpha1 $[8.5-10 \mathrm{~Hz}]$ and alpha2 [10.5-12 Hz]) and theta band power $(6.5-8 \mathrm{~Hz})$ spectra were calculated for each single epoch and then averaged across epochs.

In accordance with the study of Baumgartner et al. [6], 12 electrodes were collapsed into three frontal and three 
parietal electrode clusters: frontal left (F3/FC3), frontal midline $(\mathrm{Fz} / \mathrm{FCz})$, frontal right $(\mathrm{F} 4 / \mathrm{FC} 4)$, parietal left $(\mathrm{CP} 3 / \mathrm{P} 3)$, parietal midline $(\mathrm{CPz} / \mathrm{Pz})$, and parietal right (CP4/P4). In a first step, the two frequency band spectra (alpha and theta) were analysed separately with two four-way ANOVAs (analysis of variance) with the following repeated measurements factors: perspective (1PP vs. 3PP), agency (active vs. active), region (frontal vs. parietal) and hemisphere (left, midline and right). Greenhouse-Geisser correction was applied to guard against effects of heteroscedasticity. In a second step, dependent t-tests were used to make four pairwise comparisons between the four conditions separately for alpha and theta (1PP-active vs. 1PP-passive, $3 \mathrm{PP}$-active vs. $3 \mathrm{PP}$ passive, $1 \mathrm{PP}$-active vs. $3 \mathrm{PP}$-active and $1 \mathrm{PP}$-passive vs. 3PP-passive). The statistical analysis was performed with SPSS (version 17). The results were considered significant in case of $p<0.05$, corrected for multiple comparisons (Bonferroni). In the presentation of the ANOVA results, alpha1 and alpha2 will be reported together as alpha $(8.5-12 \mathrm{~Hz})$.

\section{sLORETA analysis}

The sLORETA software was used for source reconstruction. The sLORETA method $[32,33]$ calculates the three dimensional distribution of electrically active neuronal generators within the brain as current density values $\left(\mathrm{A} / \mathrm{cm}^{2}\right)$ based on the recorded scalp electric potential differences. sLORETA estimates the inverse problem using the assumption that the smoothest of all possible activities is the most plausible one. This assumption is supported by electrophysiological data demonstrating that neighbouring neuronal populations show highly correlated activity [34]. The sLORETA software implies a three-shell spherical head model registered to the Talairach and Tournoux atlas (Brain Imaging Centre, Montreal Neurological Institute). Source solutions space was limited to cortical gray matter and hippocampus according to the probability atlas provided by the Montreal Neurological Institute (number of voxels: 6392, voxel dimension: $5 \mathrm{~mm}^{2}$ ). It has been shown in various studies that SLORETA is able to correctly locate with fairly low errors [35-38], which has been validated from studies combining SLORETA with fMRI [39] and with PET [40]. Statistical significance is assessed by means of a nonparametric randomization test [41] and thresholds were set to $p<0.05$ (corrected for multiple comparisons).

\section{Results}

\section{Behavioural results}

Comparing SP ratings, significant main effects were found in both sub-scales (Friedman test: self-location: $\chi^{2}=25.46, d f=3, p<0.001 ;$ possible actions: $\chi^{2}=47.50$, $d f=3, p<0.001)$. Comparing the four conditions pairwise, a significant difference was found between 1PPactive and 3PP-active $(Z=2.68, p<0.01)$ and between $1 \mathrm{PP}$-passive and 3PP-passive $(Z=2.60, p<0.01)$ but not between 3PP-active vs. 1PP-passive $(Z=0.50, p>0.05)$ for self-location. Figure 2a illustrates greater SP in $1 \mathrm{PP}$ than in 3PP and this more so in the active condition than in the passive. For possible actions, the same pattern of SP ratings was observed, namely more SP in 1PP than in 3PP and more SP in the active than in the passive condition (see Figure 2b). But in this condition the comparison between 1PP-active and 3PP-active was not significant $(Z=1.57, p>0.05)$, whereas the comparisons between 3PP-active and 1PP-passive $(Z=3.71, p<0.001)$ and between 1PP-passive vs. 3PP-passive $(Z=2.16$,
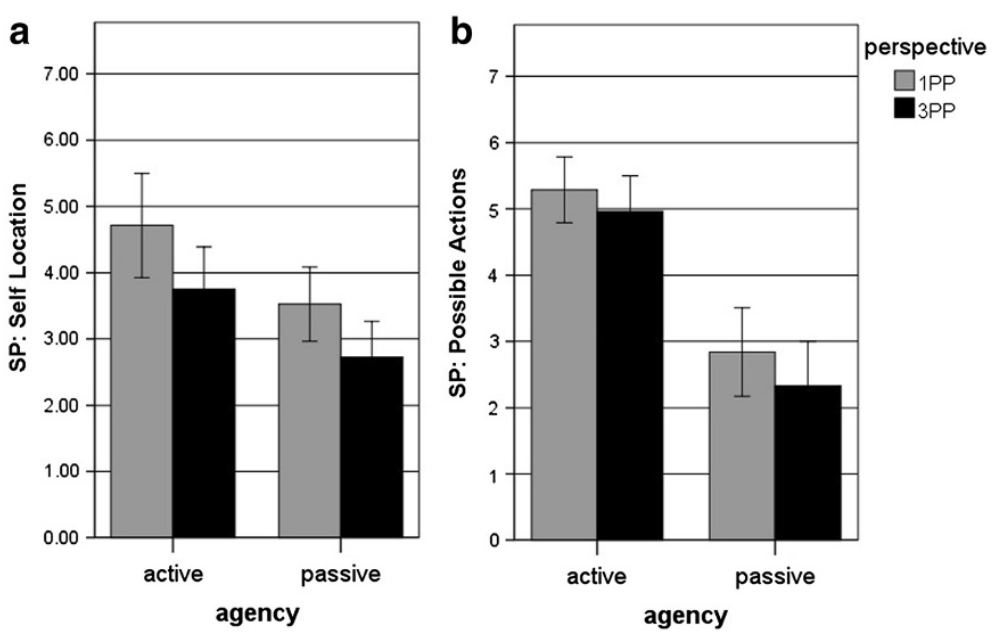

Figure 2 SP rating. Depicted are the means (with SE) in the self-location rating (Figure $2 \mathbf{a}$ ) and in the possible actions rating (Figure $2 \mathbf{b}$ ) on an 8-point scale. This is broken down for the four different conditions: 1PP-active, 3PP-active, 1PP-passive and 3PP-passive. The subjects indicated the greatest SP experience in 1PP-active, followed by 3PP-active, 1PP-passive and 3PP-passive in this order. 
$p<0.05)$ were significant. As shown in Figures $2 \mathrm{a}$ and $2 \mathrm{~b}$, agency had a larger absolute influence on both perspective conditions: Higher SP ratings were found for the active conditions (1PP-active and 3PP-active) than for the passive conditions (1PP-passive and 3PP-passive). In all of these analyses, no significant differences were found between gamers and non-gamers.

\section{Scalp map analysis}

The alpha- and theta power values were subjected to four-way ANOVAs with perspective (1PP vs. 3PP), agency (active vs. passive), region (frontal vs. parietal), and hemisphere (left, midline and right) as factors. The ANOVA for the alpha power revealed two significant main effects for agency $(F=5.306, d f=1,17, p<0.05)$ and for perspective $(F=6.841, d f=1,17, p<0.05)$ and three significant interaction effects for agency * perspective $(F=5.459, d f=1,17, p<0.05)$, for agency * hemisphere $(F=4.806, d f=2,16, p<0.05)$, and for region* hemisphere $(F=5.033, d f=2,16, p<0.05)$. The effect for agency is qualified by larger alpha band power during the passive condition compared to the active condition. The effect for perspective depends on larger alpha band power during $3 \mathrm{PP}$ than during $1 \mathrm{PP}$. The interaction between agency * perspective is qualified by larger alpha band power during $3 \mathrm{PP}$ than during $1 \mathrm{PP}$ only in the passive condition but not in the active condition. The interaction effect for agency * hemisphere demonstrated topographically the strongest alpha band power for the midline electrode clusters for the active condition and a similar distribution between the left, the midline and the right electrode clusters for the passive condition. Finally, the interaction effect for region * hemisphere revealed in frontal areas more alpha band power in the midline electrode cluster compared with the lateral electrode clusters, and in parietal areas more alpha band power in the lateral electrode clusters compared with the midline clusters. Table 1 shows the results of the post-hoc t-tests between the four conditions. In summary, and as

Table 1 post-hoc t-tests

\begin{tabular}{llllll}
\hline & & $\boldsymbol{M}$ & $\boldsymbol{t}$ & $\boldsymbol{d f}$ & $\boldsymbol{p}$ \\
\hline Alpha & 1PP-active vs. 1PP-passive & -0.12 & -1.96 & 18 & $>0.05$ \\
& 3PP-active vs. 3PP-passive & -0.17 & -2.38 & 18 & $<0.05^{*}$ \\
& 1PP-active vs. 3PP-active & 0.00 & -1.28 & 18 & $>0.05$ \\
& 1PP-passive vs. 3PP-passive & -0.05 & -2.82 & 18 & $<0.05^{*}$ \\
Theta & 1PP-active vs. 1PP-passive & 0.05 & 2.80 & 18 & $<0.05^{*}$ \\
& 3PP-active vs. 3PP-passive & 0.03 & 1.85 & 18 & $>0.05$ \\
& 1PP-active vs. 3PP-active & 0.00 & -0.41 & 18 & $>0.05$ \\
& 1PP-passive vs. 3PP-passive & -0.03 & -1.72 & 18 & $>0.05$ \\
\hline
\end{tabular}

Summarized are the results of the post-hoc t-tests between the four conditions separately for alpha and theta. The results were considered significant in case of $p<0.05$, corrected for multiple comparisons. depicted in Figures 3 and 4, there is generally more alpha band activity in the passive compared with the active condition and in the 3PP compared with 1PP.

The ANOVA for the theta band power showed three significant main effects for agency $(F=5.731, d f=1,17$, $p<0.05)$, for region $(F=39.265, d f=1,17, p<0.001)$ and for hemisphere $(F=38.521, d f=2,16, p<0.001)$, and three significant interaction effects for agency * region $(F=16.406, d f=1,17, p<0.001)$, for agency * hemisphere $(F=8.032, d f=2,16, p<0.001)$ and for region * hemisphere $(F=8.973, d f=2,16, p<0.01)$. However, there was neither a significant main effect nor a significant interaction effect for the factor perspective (main effect: $F=1.473, d f=1,17, p>0.05)$. Unlike in the alpha band ANOVA, there was more theta band power in the active condition than in the passive. In addition, the main effects for region and for hemisphere and the interaction effect for region * hemisphere showed significantly more theta band power in frontal brain parts and significantly more theta in the midline electrode clusters. The interaction effect for agency * hemisphere demonstrated increased theta band power at the midline electrode clusters for the active condition compared to the passive condition. The interaction effect for agency * region showed more theta band power in the frontal electrode clusters in the active condition compared to the passive. Table 1 shows the results of the post-hoc t-tests between the four conditions. In summary, Figures 5 and 6 illustrate that there are significant main and interaction effects for agency but not for perspective.

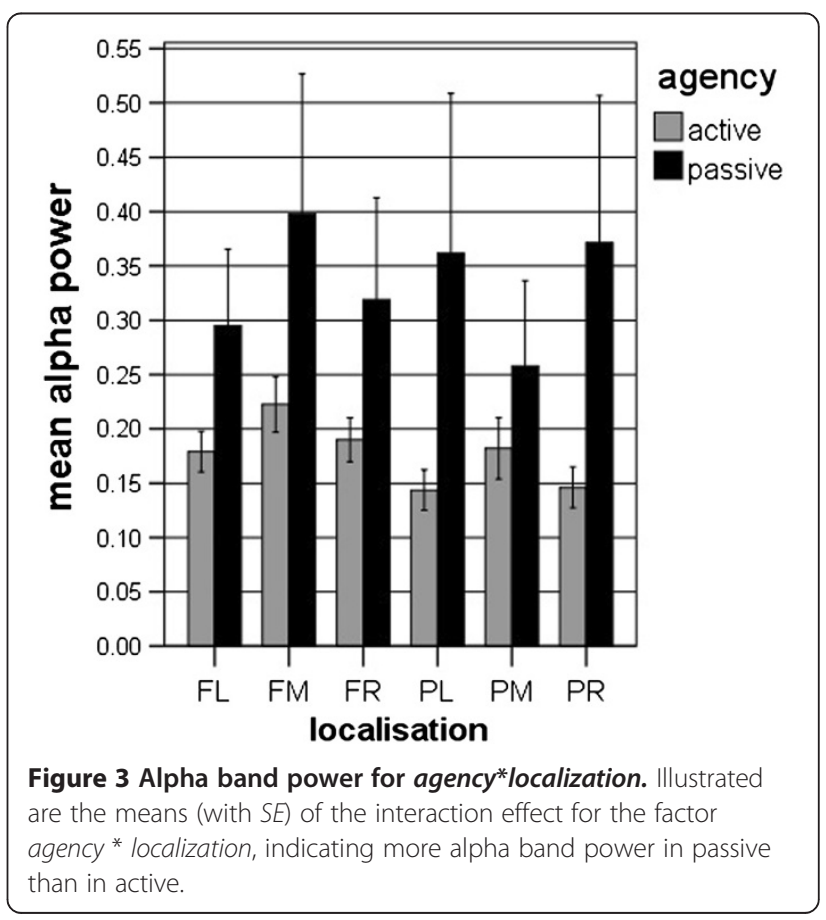




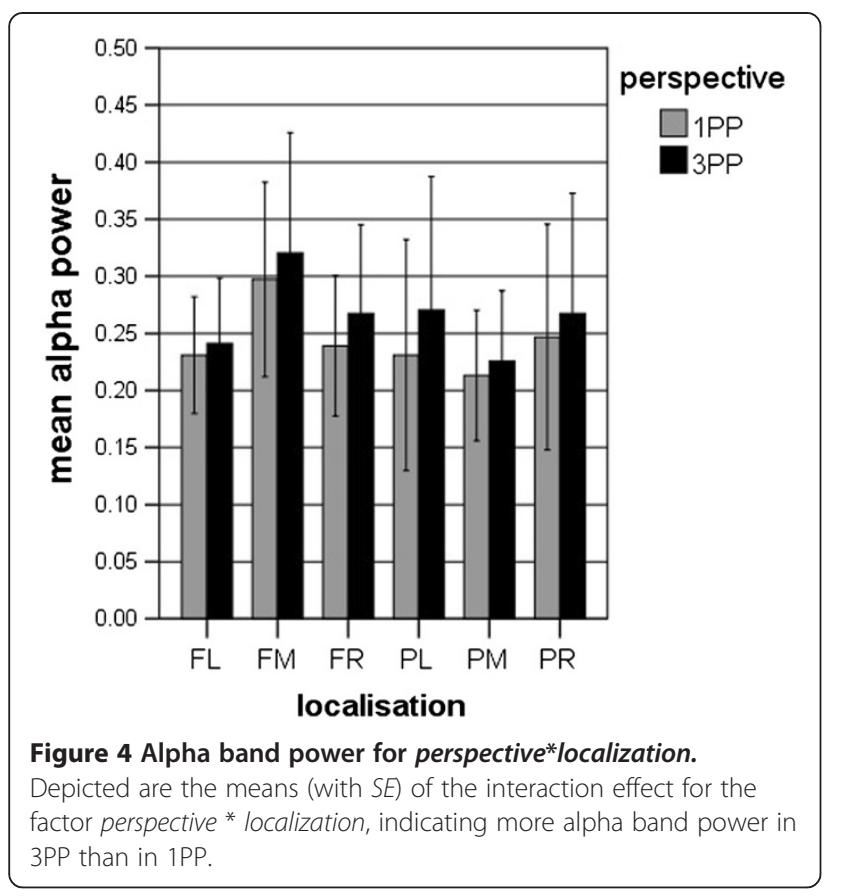

\section{SLORETA analysis}

Using SLORETA to locate the neural underpinnings (Table 2 summarizes the sLORETA results), the comparison between 1PP and 3PP (in the active condition) revealed in 1PP significant less alpha1 frequency band power in the limbic cortex (bilateral posterior cingulate, left cingulate cortex, left parahippocampalgyrus) and the occipital cortex (left lingual gyrus, left cuneus) and

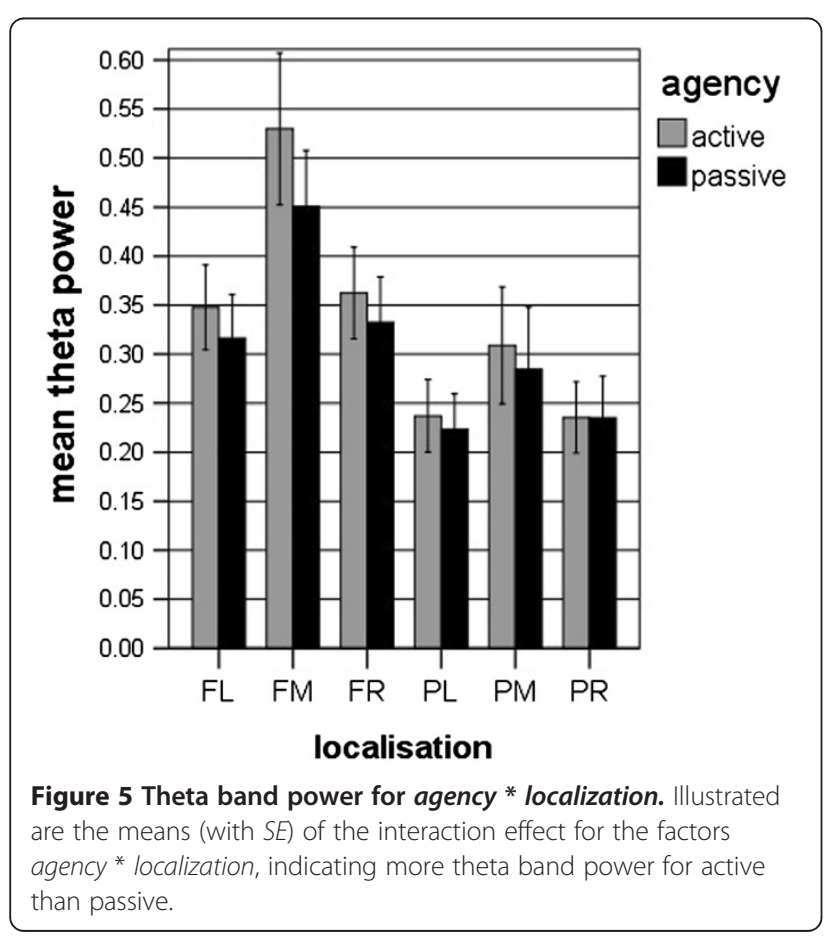

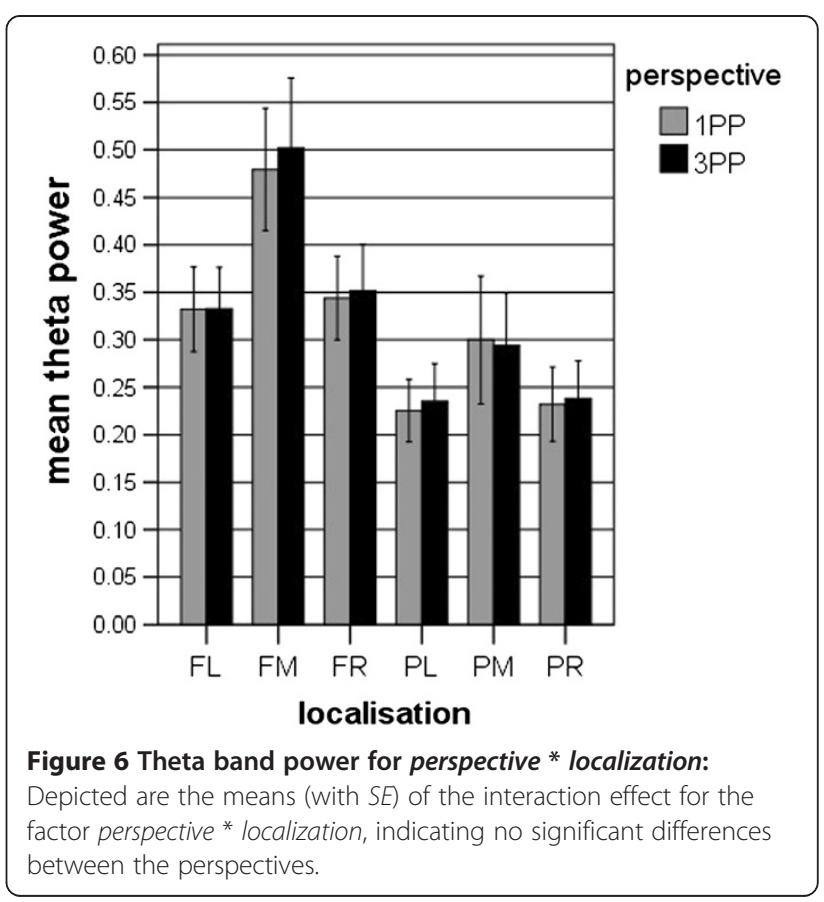

significant less alpha2 frequency band power in the parietal cortex (right inferior parietal lobule) [see Figures $7 \mathrm{a} / \mathrm{b}$ ]. The comparison between active and passive (in 1PP) revealed in the active condition compared to passive significantly more theta frequency band power in frontal brain parts bilaterally (superior, middle, medial and inferior frontal gyrus in the left hemisphere and superior and middle frontal gyrus in the right hemisphere) [see Figure 8].

To compare the present EEG study with previous EEG and fMRI studies of Baumgartner et al. [6,7], two more analyses were conducted to compare the four conditions. First, the condition that induced the highest SP experience (number of participants with the highest SP in 1PP-active: 14, 3PP-active: 3, 1PP-passive: 3 and 3PP-passive: 0 ) was compared with the condition that induced the lowest SP (number of participants with the lowest SP in 1PP-active: 1, 3PP-active: 2, 1PP-passive: 2 and 3PP-passive: 15) for each participant. This comparison showed significantly less alpha2 frequency band power in parietal brain regions of the left hemisphere (left superior and inferior parietal gyrus, left precuneus) in the high SP conditions compared with the low SP conditions [see Figure 9].

Second, a region of interest analysis for the DLPFC was performed. The mean intracerebral activation in the DLPFC of each hemisphere (left hemisphere: -30; 15; 15, right hemisphere: $30 ; 15 ; 15)$ was estimated and correlated with the SP ratings of the self-location subscale. By this, a significant correlation between SP and the deactivation in the DLPFC of both hemispheres was found: High SP ratings were correlated with the intracerebral alpha activity in both DLPFC (left hemisphere: $r_{s}=0.526$, 
Table 2 sLORETA results

\begin{tabular}{|c|c|c|c|c|c|c|c|}
\hline$x$ & $\mathrm{Y}$ & $\mathbf{Z}$ & BA & Voxel Value & Lobe & Main Structure & Cluster including \\
\hline \multicolumn{8}{|c|}{ 1PP-active vs. 1PP-passive (theta) } \\
\hline-45 & 40 & 15 & 46 & $6.34 \mathrm{E}+00$ & Frontal Lobe & Inferior Frontal Gyrus & $\begin{array}{l}\text { Superior, middle, medial, } \\
\text { inferior frontal gyrus }\end{array}$ \\
\hline 30 & 40 & 40 & 9 & $5.42 \mathrm{E}+00$ & Frontal Lobe & Middle Frontal Gyrus & Superior, middle frontal gyrus \\
\hline \multicolumn{8}{|c|}{ 1PP-active vs. 3PP-active (alpha1) } \\
\hline-10 & -50 & 5 & 29 & $-7.14 \mathrm{E}+00$ & Limbic Lobe & Posterior Cingulate & $\begin{array}{l}\text { Posterior cingulate, cingulate gyrus, } \\
\text { parahippocampal gyrus, } \\
\text { lingual gyrus, cuneus }\end{array}$ \\
\hline 5 & -50 & 5 & 29 & $-5.32 \mathrm{E}+00$ & Limbic Lobe & Posterior Cingulate & Posterior cingulate \\
\hline \multicolumn{8}{|c|}{ 1PP-active vs. 3PP-active (alpha2) } \\
\hline 35 & -65 & 40 & 39 & $-4.87 E+00$ & Parietal Lobe & Inferior Parietal Lobule & Inferior parietal lobule \\
\hline \multicolumn{8}{|c|}{ High SP vs. Low SP (alpha2) } \\
\hline-25 & -60 & 45 & 7 & $-4.06 \mathrm{E}+00$ & Parietal Lobe & Superior Parietal Lobule & Superior parietal lobule, precuneus \\
\hline
\end{tabular}

Summarized are the sLORETA results of significant differences in brain electrical activity in different comparisons and for different frequency bands. Coordinates are defined in the standard stereotaxic space of Talairach and correspond to the observed activation maxima. The row "Cluster including" describes the extent of the activated brain region. The results were considered significant in case of $p<0.05$, corrected for multiple comparisons.

$p<0.05$; right hemisphere: $r_{s}=0.566, p<0.05$; both hemispheres: $\left.r_{s}=0.563, p<0.05\right)$ [see Figure 10].

\section{Discussion}

The goal of this study was to establish whether SP is modulated by the two critical media factors perspective and agency and to delineate the neural impact of these factors during video game play. We thus conducted an experiment in which the participants played the video game "Oblivion" in four different conditions (1PP-active, 3PP-active, 1PP-passive and 3PP-passive) while scalp EEG was recorded and subjective experience of SP was assessed. The most important findings may be summarised as follows: (1) Higher SP ratings were obtained in 1PP compared with 3PP and in the active compared with the passive condition. (2) The analysis of the scalp EEG data revealed more alpha band power during 3PP than during $1 \mathrm{PP}$ and more alpha band power in the

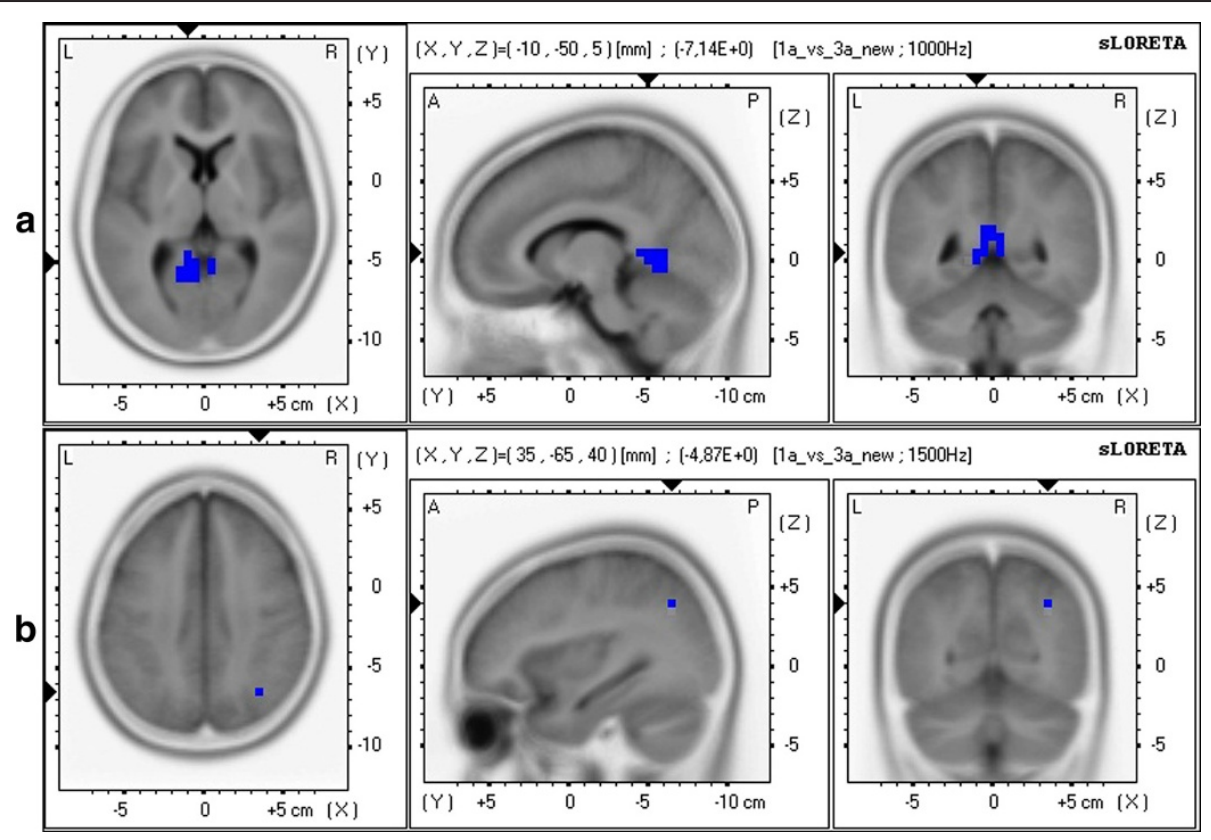

Figure 7 sLORETA images of the comparison 1PP-active vs. 3PP-active. Shown are the functional sLORETA images for the comparison 1 PP-active vs. 3PP-active in the alpha1 frequency band (Figure 7a) and alpha2 frequency band (Figure 7b). Increased activity in 1PP-active compared with the 3PP-active condition is labeled red, decreased activity is labeled blue. 


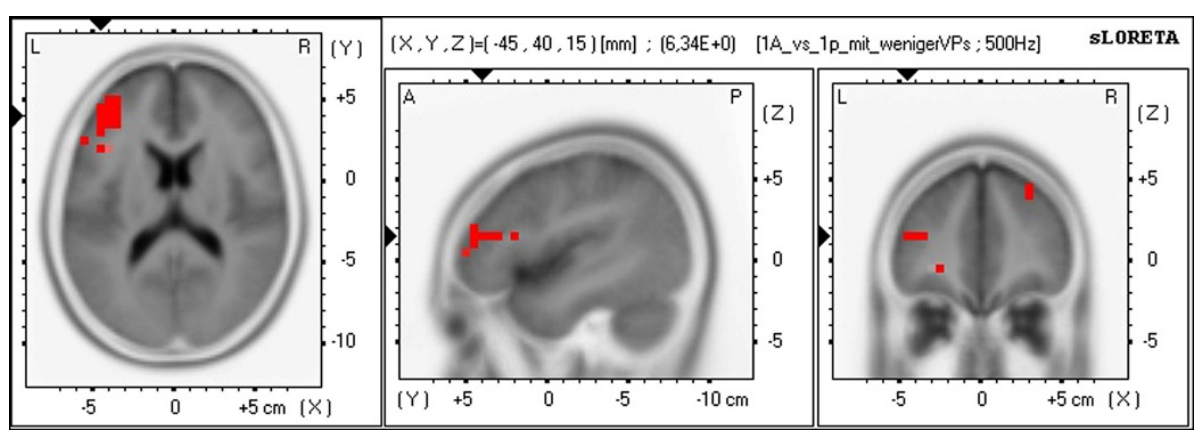

Figure 8 sLORETA images of the comparison 1PP-active vs. 1PP-passive. Shown are the functional sLORETA images for the comparison 1PP-active vs. 1PP-passive in the theta frequency band. Increased activity in 1PP-active compared with the 1PP-passive condition is labeled red, decreased activity is labeled blue.

passive than in the active condition. (3) For the theta band, there was more activity in the active than in the passive condition and no significant activity difference between the two perspectives. (4) The intracerebral activations inferred with SLORETA revealed less alpha band activity in the parietal cortex, the occipital cortex, and the limbic cortex for the 1PP compared to the 3PP condition. (5) Finally, there was stronger theta activity bilaterally in the frontal cortex including superior, middle and inferior areas in the active than in the passive condition. In the following, we will discuss these findings in the context of the literature on SP with specific emphasis on neuroscientific findings.

\section{SP feeling in the context of perspective and agency}

Operating the video game in the 1PP and active conditions was associated with the strongest subjective SP feelings, thus corroborating the results of previous studies $[12,13]$. Nevertheless, our findings go beyond these previous studies because we also examined the interaction of these two factors. Doing this, we found stronger SP ratings during the active conditions (1PP-active and in the 3PP-active) than during both passive conditions (1PP-passive and 3PP-passive), meaning that this general effect was independent of whether the subjects played in 1PP or 3PP. Thus, the factor agency exerts a stronger perception of SP than the factor perspective.

Early conceptualisations of SP have highlighted the specific role of perception on the generation of SP (the perception-oriented view). In this view, SP most strongly depends on the specific perception of spatial cues in VR. It has been argued that the person is concurrently "inattentive" to the spatial cues of the real physical surroundings [2]. Thus, a sensory-rich media stimulation evokes greater attentional processing preferentially via the visual dorsal stream and thus contributes to an enhanced perception of SP $[1,10,18]$. An alternative view of SP has been proposed by Sanchez-Vives and Slater (the action-oriented view) [4]. These authors highlight the role of controlled actions in the real or VE as a constituent feature for experiencing SP. The sense of "being there" in a VE is thought to be based on the ability to "do there". Thus, conscious action control and movement within the VE is an important constituent of SP. This does not necessarily mean that real actions must be executed in a VE. A mental representation of an action can be automatically triggered by the incoming VE stimuli irrespective of subsequent execution of the action or

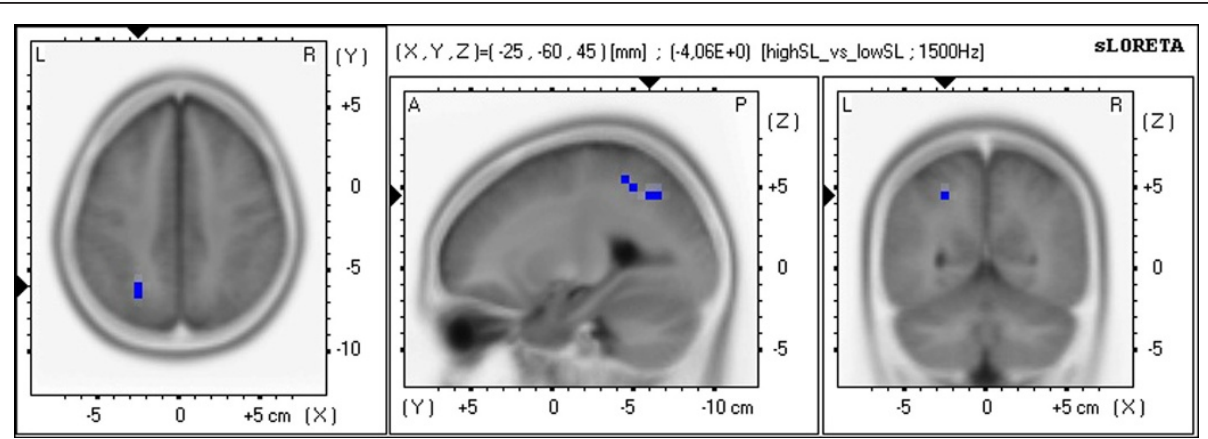

Figure 9 sLORETA images of High SP vs. Low SP. Shown are the functional SLORETA images for the comparison High SP Vs. Low SP in the alpha2 frequency band. Increased activity in High SP compared with the Low SP condition is labeled red, decreased activity is labeled blue. 


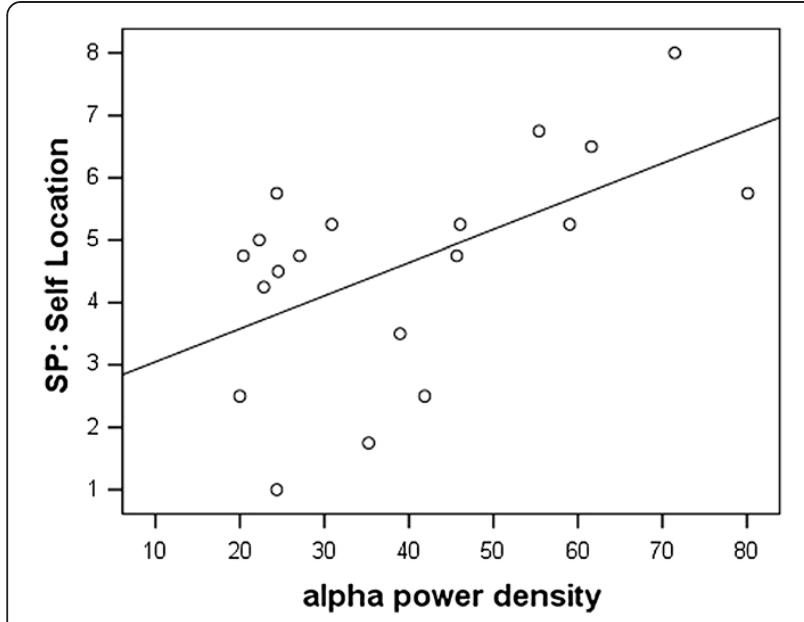

Figure 10 Correlation between SP ratings and brain activation. Depicted is the correlation between the SP rating in self-location (one dot is the value of one participant) and the mean brain activation (alpha band power in $\mathrm{mV}$ ) in the DLPFC of both hemispheres (left hemisphere: -30; 15; 15, right hemisphere: 30; 15; 15).

not [10]. Both views, the perception-oriented and the action-oriented, receive support from our own study since we demonstrate that the experience of SP can be evoked by the perceptual perspective and by the experience of agency in the VE.

However, our experiment indicates that agency is more important for SP, given the larger influence of agency than of perspective on SP ratings. This finding is consistent with the action-oriented view in a gaming situation, although this may be limited to the specific characteristics of computer and video gaming and may not be generalized to all VR situations. In a common video gaming situation like the one used in our experiment the sensory modalities are not exposed to the kind of near-real-life stimuli that more sophisticated VR technologies can deliver. Sophisticated VR technologies are capable of addressing different sensory modalities and of sustaining a match between these different modalities (e.g. by using eye goggles and real-time tracking devices) [42]. However, in video or computer gaming, the means to stimulate sensory modalities are technically limited to a TV or computer screen. But we suggest that in video gaming this limitation can be compensated for by an increase in the interactivity enabled or required by the mediated environment. Referring to the model of Wirth et al. [5], we postulate that the possibility to act and manipulate first "attracts" the attention of the player to the VE and, in a second step, increases the acceptance of the mediated ERF as the primary ERF. Consequently, it is possible that (especially in video gaming) the perception of agency is of higher importance than the perception of self-location.

\section{Neurophysiology in the context of perspective and agency}

With respect to the cortical activation pattern, we identified decreased alpha band activity in the active and 1PP conditions. Since the active and 1PP conditions evoke the strongest SP perception, it is clear that strong SP is associated with less alpha band activity. Based on studies that revealed strong negative relationships between hemodynamic responses and alpha band power [24-26], we take the alpha band power as an indicator of cortical deactivation in these areas. Thus, higher cortical activation (strong hemodynamic responses) is associated with less alpha band activity. In the context of our study, the weaker alpha band responses over frontal and over parietal areas during $1 \mathrm{PP}$ and the active conditions indicates increased brain activation during these conditions. Since these conditions are also those with the strongest sense of SP, we assume that strong SP is associated with increased activation in the fronto-parietal network. For the theta band, we identified more theta band power (especially over the frontal midline positions) during the active conditions. In contrast to alpha band activity, theta band activity is not a direct indicator of neural activation or deactivation. However, theta band activity, especially over the frontal midline electrodes (FM-theta), is known to be involved in the control of complex cognitive operations such as working memory, memory processes, and the control of actions [27,28]. Furthermore, our finding of increased FM-theta in active conditions is consistent with previous EEG based video gaming studies in showing that operating actions in VEs is associated with increased FM-theta [29-31].

\section{Intracerebral activations in the context of SP feeling}

In the comparison between high SP conditions and low SP conditions, we found less alpha2 frequency band power in parietal brain regions of the left hemisphere (left superior and inferior parietal gyrus, left precuneus) in the high SP conditions. Additionally, we revealed a significant positive correlation between intracerebral alpha activity in the bilateral DLPFC and SP ratings of the self-location subscale. These findings are in accordance with previous findings of our group in which we demonstrated 1) that SP experience is associated with the involvement of a distributed fronto-parietal network including the dorsal visual stream as a prominent part, and 2) that the DLPFC is one of the major regulators within this network [6,7]. Nevertheless, our results not only replicate previous findings but expand current knowledge by showing that these neurophysiological mechanisms are not confined to the virtual roller coaster scenarios used by Baumgartner et al. [6,7]. Since the roller coaster scenarios were non-interactive and arousing, while our experimental scenarios were interactive 
and non-arousing, we demonstrate that the core regions associated with SP are relevant in different virtual environments.

\section{Intracerebral activations in the context of perspective and agency}

Comparison of the intracerebral sources of alpha activity between 1PP and 3PP (separately for the active and the passive conditions) revealed a network of neural sources including the parietal cortex (right inferior parietal lobule), the occipital cortex (left lingual gyrus, left cuneus), and the limbic cortex (bilateral posterior cingulate, left cingulate cortex, left parahippocampalgyrus) with decreased alpha activity. Comparison of the active and passive conditions showed stronger theta activity in frontal brain areas during the active conditions (superior, middle, medial and inferior frontal gyrus in the left hemisphere, and superior and middle frontal gyrus in the right hemisphere). The fronto-parietal network found in the present study (and in previous studies of our group) is not exclusively associated with generating and controlling the experience of SP [see also [10]. These regions of the parietal cortex are known to be involved in spatial processing, mental rotation, and most importantly, a part of this network constitutes the dorsal visual stream, which is known to be involved in the egocentric processing $[43,44]$. These parts of the limbic cortex are known to play an important role in episodic memory, emotional stimuli processing, and action control $[23,35]$. The identified frontal brain areas are known to be involved in various executive functions that exert regulatory control over emotions and actions with the DLPFC being an important part [27,45-51].

Among the various brain regions within this frontoparietal network, the role of a parietal-premotor connection associated with sensory-motor integration is particularly interesting for the interpretation of our findings for perspective and agency. The posterior parietal cortex (PPC) is known to integrate information from different sensory modalities to form a coherent multimodal representation of space coded in a body-centered reference frame. This integration of multisensory cues includes mapping the position of objects in reference to one's own body [52]. The premotor cortex, (PMC) on the other hand, appears to define a corresponding motor space consisting of all potential motor actions within the surrounding space [53]. It has been suggested that potential target objects within the visual field elicit motor schemas for potential actions that map the position of these objects in the surrounding environment [54]. Put simply, the PPC directly maps the position of objects in reference to the body, whereas the PMC creates a motor space as an intermediate step first.
According to the model of Wirth et al. [5], an alternative egocentric reference frame (ERF) to the real-world ERF develops from spatial cues and action cues of the VE. This ERF contains information about the spatial properties of immediate surroundings from a firstperson perspective. The parietal-premotor connection might play a key role in this process. As mentioned in a previous review [10], we believe that the new egocentric view derived from VR is generated in the context of several transformation processes in the dorsal visual stream of the parietal cortex [see [43,44]]. Regarding our findings for perspective and agency, we propose that mediated by the PPC, spatial cues from the VE directly define the spatial properties within an egocentric view. On the other hand, mediated by the PMC, actions cues in the VE are used to define a motor space which indirectly facilitates an egocentric view. Thus, we propose that both parietal and frontal regions are involved in the generation of the alternative ERF derived from the mediated environment.

The sense of SP is thought to emerge when the ERF from the mediated environment is selected as the primary ERF over the competing ERF of the actual environment (Wirth et al. [5]). We believe that whether or not a person chooses this new ERF as the primary ERF depends (among other things) upon the strength of the influence that media factors such as perspective and agency exert on this fronto-parietal network. More specifically, we propose that playing in $1 \mathrm{PP}$, as well as actively controlling the actions of the video game avatar, increases the acceptance of the new ERF as the primary ERF mediated by (and observable as) increased activation in the fronto-parietal network.

\section{Limitations}

The present study has some limitations which should be addressed in future studies. The SP model of Wirth et al. [5], which lays the theoretical foundation for the interpretation of our neuroscientific findings, has not yet been thoroughly investigated empirically. It remains to be seen if this model will receive further support in future investigations.

The post-hoc questionnaires used in this study have disadvantages due to their administration after exposure; these disadvantages include recency effects, anchoring effects and inaccurate recall [55]. However, the alternative of a continuous on-line measurement of SP also has important disadvantages such as disruption of the SP experience itself by drawing attention away from the VE [2]. Future studies may be able to use new developed measurement procedures which seek to overcome these issues.

A limitation of our study design was the fact that we were not able to investigate the factors perspective and 
agency independently. In future studies, it may prove beneficial to manipulate the amount of sensory information and the possibilities to act in the VE independently in order to further elaborate how each influence SP and which influences SP more significantly.

\section{Conclusion}

In sum, we propose that manipulating the factors perspective and agency influences SP by either directly or indirectly modulating the ego-centric visual processing in a fronto-parietal network. The findings of this study fit well within recent neuroimaging and behaviour studies. Several studies have demonstrated that a strong sense of SP is associated with activation in a distributed brain network that specifically includes fronto-parietal regions [6-10]. But our findings are also consistent with different theoretical views on presence (i.e. action- and perception-oriented) and may thus help to reconcile these views in one conceptual framework $[5,10]$.

\section{Competing interests}

The authors declare that they have no competing interests.

\section{Authors' contributions}

$\mathrm{MH}$ conducted the literature review, planned the experimental design, performed data collection and data analysis, and prepared all drafts of the manuscript. NL contributed to data analysis and to draft revision. MC contributed to planning the experimental design, contributed to data collection and to draft revision. $\sqcup$ contributed to draft revision and to theoretical interpretation. All authors have read and approved the final manuscript.

\section{Acknowledgements}

This work was supported by the European Union FET Integrated Project PRESENCCIA (Contract number 27731). Furthermore, we would like to acknowledge Janine Saurer for her help in data collection.

\section{Author details}

${ }^{1}$ Clinic for Affective Disorders, University Clinic of Psychiatry Zurich, Zurich, Switzerland. ${ }^{2}$ Department of Neuropsychology, Psychological Institute, University of Zurich, Zurich, Switzerland.

Received: 2 November 2011 Accepted: 29 June 2012 Published: 19 July 2012

\section{References}

1. Witmer $B$, Singer M: Measuring presence in virtual environments: $A$ presence questionnaire. Presence-teleop Virt 1998, 7:266-267.

2. ljsselsteinjn $W$, de Riddler $H$, et al: Presence: Concept, determinants and measurement. P Soc Photo-opt Ins 2000, 3959:520-529.

3. Slater M: Presence and emotions. Cyberpsychol Behav 2004, 7:121. author reply 123 .

4. Sanchez-Vives M, Slater M: From presence to consciousness through virtual reality. Nat Rev Neurosci 2005, 6:332-339.

5. Wirth $W$, Hatmann $T$, et al: A process model of the formation of spatial presence experiences. Media Psychol 2007, 9:493-525.

6. Baumgarten $T$, Valko $L$, et al: Neural correlate of spatial presence in an arousing and noninteractive virtual reality: an EEG and psychophysiology study. Cyberpsychol Behav 2006, 9:30-45

7. Baumgartner $T$, Speck D, et al: Feeling present in arousing virtual reality worlds: prefrontal brain regions differentially orchestrate presence experience in adults and children. Front Hum Neurosci 2008, 2:8.
8. Jancke $L$, Brunner $B$, et al: Brain activation during fast driving in a driving simulator: the role of the lateral prefrontal cortex. Neuroreport 2008, 19:1127-1130.

9. Beeli G, Casutt $G$, et al: Modulating presence and impulsiveness by external stimulation of the brain. Behav Brain Funct 2008, 4:33.

10. Jancke $L$, Cheetham $M$, et al: Virtual reality and the role of the prefrontal cortex in adults and children. Front Neurosci 2009, 3:52-59.

11. Klasen $M$, Weber $R$, et al: Neural contributions to flow experience during video game playing. Soc Cogn Affect Neurosci 2012, 7:485-495.

12. Larsson $P$, Vastfjall $D$, et al: The actor-observer effect in virtual reality presentations. Cyberpsychol Behav 2001, 4:239-246.

13. Kallinen $\mathrm{K}$, Salminen $M$, et al: Presence and emotion in computer game players during 1st person vs. 3rd person playing view: Evidence from self-report, eye-tracking, and facial muscle activity data. Proceedings of the PRESENCE 2007, 187-190

14. Salminen M, Ravaja N: Oscillatory brain responses evoked by video game events: the case of super monkey ball 2. Cyberpsychol Behav 2007, 10:330-338.

15. Salminen M, Ravaja N: Increased oscillatory theta activation evoked by violent digital game events. Neurosci Lett 2008, 435:69-72.

16. Pascual-Marqui RD: Standardized low-resolution brain electromagnetic tomography (sLORETA): technical details. Methods Find Exp Clin Pharmacol 2002, 24(suppl D):5-12.

17. Annett M: A classification of hand preference by association analysis. $\mathrm{Br} \mathrm{J}$ Psychol 1970, 61:303-321.

18. Vorderer P, Wirth W, et al: MEC spatial presence questionnaire (MEC-SPQ): Short documentation and instructions for application. Report to the European Community, Project Presence: MEC. ISTth edition, 2001:37661.

19. Lehmann D: The view of an EEG-EP mapper. Brain Topogr 1988, 1:77-78.

20. Lehmann D: Past, present and future of topographic mapping. Brain Topogr 1990, 3:191-202.

21. Jung TP, Makeig S, et al: Independent component analysis of electroencephalographic and event-related potential data. In Auditory processing and neural modelling. Edited by Poon P, Brugge J. New York: Plenum Press; 1998:189-197.

22. Lehmann D, Skrandies W: Reference-free identification of components of checkerboard-evoked multichannel potential fields. ElectroencephalogrClin Neurophysiol 1980, 48:609-621.

23. Kubicki S, Herrmann WM: Reflections on the topics: EEG frequency bands and regulation of vigilance. Pharmakopsychiatr Neuropsychopharmako.l 1979, 12:237-245.

24. Laufs $H$, Krakow K: Electroencephalographic signatures of attentional and cognitive default modes in spontaneous brain activity fluctuations at rest. ProcNatl Acad Sci. USA 2003, 100:11053-11058.

25. Laufs $H$, Kleinschmidt $A$, et al: EEG-correlated $f M R I$ of human alpha activity. Neurolmage 2003, 19:1463-1476.

26. Gamma A, Lehmann $D$, et al: Comparison of simultaneously recorded $[\mathrm{H} 2$ (15)O-PET and LORETA during cognitive and pharmacological activation. Hum Brain Mapp 2004, 22:83-96.

27. Luu P, Tucker DM, Makeig S: Frontal midline theta and the err-related negativity: neurophysiological mechanisms of action regulation. Clin Neurophysiol 2004, 115:1821-1835.

28. Mitchell DJ, McNaughton N, Flanagan D, Kirk IJ: Frontal-midline theta from the perspective of hippocampal "theta". Prog Neurobio/ 2008, 86:156-185.

29. Yamada F: Frontal midline theta rhythm and eyeblinking activity during a VDT task and a video game: useful tools for psychophysiology in ergonomics. Ergonomics 1998, 41:678-688.

30. Smith ME, Gevins A, et al: Monitoring task loading with multivariate EEG measures during complex forms of human-computer interaction. Hum Factors 2001, 43:366-380.

31. Pellouchoud E, Smith ME, et al: Mental effort-related EEG modulation during video game play: comparison between juvenile subjects with epilepsy and normal control subjects. Epilepsia 1999, 40:38-43.

32. Pascual-Marqui RD, Michel CM, et al: Low-resolution electromagnetic tomography: a new method for localizing electrical activity in the brain. Int J Psychophysiol 1994, 18:49-65.

33. Pasqual-Marqui RD, Lehmann D, et al: Low-resolution brain electromagnetic tomography (LORETA) functional imaging in acute, neuroleptic-naive, first-episode, productive schizophrenia. Psychiatry Res 1999, 90:169-179. 
34. Haalman I, Vaadia E: Dynamics of neuronal interactions: relation to behavior, firing rates, and distance between neurons. Hum Brain Mapp 1997, 5:249-253.

35. Menendez RGS, Andino SG, et al: Noninvasive localization of electromagnetic epileptic activity. I. Method descriptions and simulations. Brain Topogr 2001, 14:131-137.

36. Pasqual-Marqui RD, Esslen $M$, et al: Functional imaging with low-resolution brain electromagnetic tomography (LORETA): a review. Methods Find Exp Clin Pharmacol 2002, 24:91-95.

37. Phillips $C$, Rugg $M D$, et al: Anatomically informed basis functions for $E E G$ source localization: combining functional and anatomical constrains. Neurolmage 2002, 16:678-695.

38. Esslen M, Pascual-Marqui RD, et al: Brain areas and time course of emotional processing. Neurolmage 2004, 21:1189-1203.

39. Vittacco D, Brandeis $D$, et al: Correspondence of event-related potential tomography and functional magnetic resonance imaging during language processing. Hum Brain Mapp 2002, 17:4-12.

40. Zumsteg D, Wennberg RA, et al: $\mathrm{H} 2(15) \mathrm{O}$ or $13 \mathrm{NH} 3 \mathrm{PET}$ and electromagnetic tomography (LORETA) during partial status epilepticus. Neurology 2005, 65:1657-1660.

41. Nichols TE, Holmes AP: Nonparametric Permutation Tests for Functional Neuroimaging: A Primer with Examples. Hum Brain Mapp 2002, 15:1-25.

42. Biocca F, Delany B: Immersive virtual reality technology. In Communication in the age of virtual reality. Edited by Biocca F, Levy M. Hillsdale, NJ: Lawrence Erlbaum; 1995:15-32.

43. Gron $G$, Wunderlich AP, et al: Brain activation during human navigation: gender-different neural networks as substrate of performance. National Neuroscience 2000, 3:404-408.

44. Jordan K, Schadow J, et al: Different cortical activations for subjects using allocentric or egocentric strategies in a virtual navigation task. Neuroreport 2004, 15:135-140.

45. Koechlin E, Ody C, et al: The architecture of cognitive control in the human prefrontal cortex. Science 2003, 302:1181-1185.

46. Ridderinkhof KR, Ullsperger $M$, et al: The role of the medial frontal cortex in cognitive control. Science 2004, 306:443-447.

47. Knoch D, Gianotti LR, et al: Disruption of right prefrontal cortex by lowfrequency repetitive transcranial magnetic stimulation induces risktaking behavio. J Neurosci 2006, 26:6469-6472.

48. Knoch D, Pascual-Leone A, et al: Diminishing reciprocal fairness by disrupting the right prefrontal cortex. Science 2006, 314:829-832.

49. Koechlin E, Hyafil A: Anterior prefrontal function and the limits of human decision-making. Science 2007, 318:594-598.

50. Wood JN, Grafman J: Human prefrontal cortex: Processing and representational perspectives. Nat Rev Neurosci 2003, 4:139-147.

51. Knoch D: Funktionelle Hemispharenasymmetrie der selbstkontrolle. Z Neuropsychol 2007, 18:183-192.

52. Makin TR, Holmes NP, et al: Is that near my hand? Multisensory representation of peripersonal space in human intraparietal sulcus. J Neurosci 2007, 27:731-740.

53. Gallese V: Embodied simulation: from neurons to phenomenal experience. Phenomenol Cogn Sci 2005, 4:22-48.

54. Jeannerod M, Arbib MA, et al: Grasping objects: the cortical mechanisms of visuomotor transformation. Trends Neurosci 1995, 18:314-320.

55. van Baren J, IJsselsteijn WA: Measuring presence: A guide to current measurement approaches. Deliverable 5. ISTth edition.: FET OMNIPRES Project; 2004

doi:10.1186/1744-9081-8-34

Cite this article as: Havranek et al:: Perspective and agency during video gaming influences spatial presence experience and brain activation patterns. Behavioral and Brain Functions 2012 8:34.

\section{Submit your next manuscript to BioMed Central and take full advantage of:}

- Convenient online submission

- Thorough peer review

- No space constraints or color figure charges

- Immediate publication on acceptance

- Inclusion in PubMed, CAS, Scopus and Google Scholar

- Research which is freely available for redistribution

Submit your manuscript at www.biomedcentral.com/submit
C Biomed Central 